IP Periodica Polytechnica

Transportation Engineering

44(2), pp. 115-122, 2016

DOI: $10.3311 /$ PPtr. 8547

Creative Commons Attribution (i)

RESEARCH ARTICLE

\section{Simulation Support in Construction Uncertainty Management: a Production Modelling Approach}

\author{
András Pfeiffer ${ }^{1}$, Botond Kádár ${ }^{1}$, Gábor Bohács ${ }^{2 *}$, Dániel Gáspár ${ }^{2}$
}

Received 06 September 2015; accepted 07 October 2015

\begin{abstract}
The execution of construction projects such as a highway construction or the elevation of a new bridge is a complex, highly equipment-intensive process and are subject to many different uncertainties. This is very similar to the manufacturing execution level in production systems where predefined productions plans and schedules cannot be completely implemented due to unexpected internal and external changes and disturbances. Following this analogy, the paper proposes the application of a discrete-event simulation based method which was already applied in the decision-support for manufacturing control to develop the decision-support in the execution of a construction project where the effects of the deviation from the short-term schedule can be easily and quickly analyzed.
\end{abstract}

\section{Keywords}

Construction project, simulation, planning and scheduling, disturbance handling, uncertainty management

\footnotetext{
${ }^{1}$ Fraunhofer Project Center on Production Management and Informatics, Computer and Automation Research Institute, Hungarian Academy of Sciences, Kende u. 13-17, H-1111 Budapest, Hungary

${ }^{2}$ Department of Materials Handling and Logistics Systems, Faculty of Transportation Engineering and Vehicle Engineering, Budapest University of Technology and Economics, Mủegyetem rkp. 3, H-1111 Budapest, Hungary

${ }^{*}$ Corresponding author, e-mail: gabor.bohacs@logisztika.bme.hu
}

\section{Introduction}

The execution of construction projects such as a highway construction or the elevation of a new bridge is a complex, highly equipment-intensive process and are subject to many different uncertainties (Motawa et al., 2007). Capacity and availability of machinery and human resources, material supply, technology and precedence constraints, geographical and weather conditions are all important aspect in both planning and the execution of a construction project. Planning and scheduling of such projects are done a-priori taking into account the relevant resources, preliminary set-up activities, time, space and precedence constraints, etc. (Hinze, 2011). Nowadays, several different approaches and tools are applied to generate the longer-term plan and shorter-term, detailed schedule(s) of a construction project. The schedules which cover shorter periods like several days or weeks are subject to changes thus new and new schedules are generated on given-period basis (e.g. weekly) taking the finished operations and the new tasks to be performed into consideration. The need for change in the schedule the so-called rescheduling comes from the field manager's level where the monitoring and controlling of real-time construction activities are carried out. The milestones of the project are always kept in the longer-term plan and these plans are only changed if significant delays occur, which danger the achievement of milestones and success of the construction.

The aspects enumerated above are analogous with the characteristics of production planning and scheduling and manufacturing shop-floor control, especially of those in project-base manufacturing. In the paper we propose the application of a discrete-event simulation based method which was already applied in the decision-support for manufacturing control. The method addresses the decision-support in the execution of a construction project where the effects of the deviation from the short-term schedule can be easily and quickly analysed.

\section{Handling changes in construction projects 2.1 Types of changes and disturbances}

Hao (2008) gives a comprehensive and well-classified summary of changes and disturbances in construction projects and 
they use the definition of changes in construction projects as defined by Motawa et al. (2007). We take this classification as reference in our approach and the method presented in the paper tackles the construction execution level. The summary table in the paper of Hao (2008) classifies the changes according to their stage e.g. specification, design and construction execution, and specifies the types of possible changes, their impacts and the necessary actions for each stage. Here we will only focus on the types of changes of the execution stage where the most common problems occur because of uncertainties and disturbances in:

- unavailability of planned resources and materials,

- change in the weather conditions,

- quality defects in the applied materials,

- unexpected site conditions, which were not considered in the design phase,

- deterioration, non-conformity with the design, which was not observed earlier.

Some of the problems enumerated above may cause changes in the design so that there might be no option to continue the construction. Such big impacts are not considered in our approach we will only focus on the cases where alternative options are available and new schedules for the next execution period can be generated.

\subsection{Dealing with changes - project execution control}

As it was defined previously, a manufacturing system organizes equipment, personnel, and information to create products that are delivered to a customer, and thus satisfying customer demands. This system may be as large as a factory or as small as a manufacturing cell.

Scheduling activities involve allocation of resources to the operations of multiple independent processes over time in order to achieve a targeted global behaviour (Smith, 1994). Examples are the coordination of production in a factory, or transportation scheduling. In order to be viable as operational guidance, a schedule (solution) must first be feasible, i.e., it must satisfy the physical constraints in the field relating to usage of resources and execution of processes. In practice - regarding the character of these constraints - these are often wide ranging and complex.

In manufacturing production environments, for example, resource allocation decisions must be consistent with capacity limitations, machine setup requirements, batching constraints on parallel use work shift times, etc. (Pinedo, 2002). Similarly, production activities have predefined duration and precedence constraints and may require the availability of multiple resources (e.g., machines, operators, tooling, raw materials).

In order to control production in dynamic scheduling environments where parameters are uncertain, two common strategies are known, first, predictive-reactive scheduling techniques and second, dynamic scheduling solutions (on-line or closed-loop scheduling).
The predictive-reactive approach means calculating a predictive (off-line or open-loop) schedule concerning a static problem, and continuously updating this existing schedule in order to adapt schedules to changing circumstances (reactive this way).

The process of modifying the predictive schedule against execution disruptions (internal disruptions) is referred to as reactive scheduling or rescheduling. Expressions for predictive schedules before the schedule modification (schedule revision) are quite different: original, initial, baseline or preschedule are notations commonly used in several papers.

A rescheduling policy specifies when and how rescheduling is done. The policy specifies the events that cause rescheduling. These events may be predictable (even regular) or unpredictable. The policy specifies the method used for revising the existing schedule. Note that the policy may specify different methods for different situations. If these policies have any parameters (for instance, the length of the rescheduling period), the policy specifies these parameters. Rescheduling methods generate and update production schedules.

In the coming space, a brief outline of the terms and definitions used for production scheduling as well as for construction project execution are described.

\section{Simulation support in construction}

In the following part the role of simulation-based production schedule evaluation - as an analogue to construction project scheduling -, as well a new approach is described including the proposed structure, the model building and the construction site modelling features.

\subsection{Schedule evaluation by using simulation in production}

The discrete-event simulation (hereafter referred to as simulation) approach has been widely applied to decisions in planning and scheduling, related to production applications (see e.g., (Banks, 1998; Law and Kelton, 2000; O’Reilly, 1999)). The simulation models that are used for making or evaluating these decisions generally represent the flow of materials to and from processing machines and the operations of machines themselves (Rabelo et al., 2003). Potential problems can be identified and can be corrected using a simulation model. By far the most common use of simulation models is for operational decisions such as planning and scheduling (Law and Kelton, 2000).

Simulation captures those relevant aspects of the production planning and control (PPC) problem which cannot be represented in a deterministic, constraint-based optimization model. The most important issues in this respect are uncertain availability of resources, uncertain processing times, uncertain quality of raw materials, and insertion of conditional operations into the technological routings. Aytug et al. (2005) give a broad overview in their study on production schedule execution in the face of uncertainties. Categorization of uncertainty 
Table 1 Mapping terms applied in manufacturing industry to construction industry

\begin{tabular}{|c|c|c|}
\hline Term & Production scheduling & Construction project scheduling \\
\hline $\begin{array}{l}\text { Operational } \\
\text { control }\end{array}$ & $\begin{array}{l}\text { Shop floor control determines which operation each person and piece of } \\
\text { equipment should perform and when they should do it. In general this } \\
\text { activity controls all manufacturing and material handling resources. }\end{array}$ & $\begin{array}{l}\text { Field manager level control with similar goals as shop floor } \\
\text { control. }\end{array}$ \\
\hline Schedule & $\begin{array}{l}\text { A production schedule specifies, for each resource required for } \\
\text { operations, the planned start time and end time of each operation } \\
\text { assigned to that resource. }\end{array}$ & $\begin{array}{l}\text { Project schedule is a collection of activities that are } \\
\text { aggregated from the detailed operation plans of the projects, } \\
\text { and allocates tasks }\end{array}$ \\
\hline $\begin{array}{l}\text { Task, activity, } \\
\text { operation }\end{array}$ & $\begin{array}{l}\text { Operation means the execution of a process (such as machining, form- } \\
\text { ing, assembly, testing and inspection) on a given item by using a certain } \\
\text { set of resources. }\end{array}$ & $\begin{array}{l}\text { Task is an activity or a collection of activities which has/ } \\
\text { have to be completed within a time range. }\end{array}$ \\
\hline Order release & $\begin{array}{l}\text { Order release controls a manufacturing system's input by determining } \\
\text { which orders (jobs) should be moved into production (job release, order } \\
\text { review/release). }\end{array}$ & Release of the different projects/subproject \\
\hline WIP control & $\begin{array}{l}\text { Control method for order release, e.g., controlling the level of production } \\
\text { orders at a time in different production segments of a plant }\end{array}$ & $\begin{array}{l}\text { Controlling the number of parallel running tasks at a given } \\
\text { location/site, considering e.g., regulations, load constraints. }\end{array}$ \\
\hline Intensity & $\begin{array}{l}\text { Varying level of capacity constrains regards to resource availability. } \\
\text { Mainly defined on a shift level, e.g., during the night shift less operators } \\
\text { are available on an assembly line. }\end{array}$ & $\begin{array}{l}\text { Typical behaviour of construction scheduling problems; } \\
\text { projects/subprojects have ramp-up and ramp-down phases } \\
\text { where the intensity of activities/tasks are lower, however } \\
\text { in some phases of the project increased intensity requires } \\
\text { additional resources (outsourcing). }\end{array}$ \\
\hline $\begin{array}{l}\text { Resource } \\
\text { (renewable }\end{array}$ & tool & attachment \\
\hline $\begin{array}{l}\text { Resource } \\
\text { (non-renewable) }\end{array}$ & raw material & construction materials \\
\hline Location & $\begin{array}{l}\text { Usually factory, plant, segment, cell, machine levels are considered., in } \\
\text { which resources are available }\end{array}$ & $\begin{array}{l}\text { Various locations (geographically, e.g., construction site) } \\
\text { where tasks are allocated (i.e., mainly assigned to locations) } \\
\text { and have to be processed by certain resources. }\end{array}$ \\
\hline $\begin{array}{l}\text { Spatial con- } \\
\text { straints }\end{array}$ & Buffer levels, storage capacity constraints & $\begin{array}{l}\text { Spatial constraints of the site, e.g., limited space available } \\
\text { for assembly of components. }\end{array}$ \\
\hline Horizon & shift/daily/weekly & daily/weekly \\
\hline Process & milling drilling turning assembly welding & $\begin{array}{l}\text { earthwork, concrete work, reinforcement work, electric } \\
\text { installation }\end{array}$ \\
\hline $\begin{array}{l}\text { Precedence } \\
\text { constraint }\end{array}$ & Precedence order other tasks and activities & Precedence order other tasks and activities \\
\hline
\end{tabular}

is formulated for a better understanding of the meaning of uncertainty during the calculation or execution of a production plan or schedule.

In simulation supported schedule evaluation, simulation is often used for evaluating the robustness of the schedule. A production schedule is termed robust in case it performs well after a disruption. The usefulness of simulations lies in detecting and preventing these problems concerning robustness before the detailed, short-term schedule reaches the shop floor. Thus, the key benefit of a simulation-based schedule evaluation system is the feedback about schedule performance (e.g., number of late tasks) which, in turn, can be used for improving subsequent solutions. Recently applied schedule evaluation methods of (predictive) production schedules during simulation-based assessment are classified in the paper of Kempf et al. (2000) regarding the environment of the evaluation (static/dynamic) and the evaluation criteria of the schedules (absolute/relative).

A number of authors present simulation-based experimental studies with the aim at analysing scheduling problems and schedule evaluation techniques in a dynamic and stochastic environment. The categorization of the selected papers is also highlighted in the paper of Pfeiffer et al. (2007). The analytical solutions proposed in (Pfeiffer et al., 2007) are able to estimate important performance measures for schedule evaluation methods in a dynamic, stochastic manufacturing system, and are evaluated in simulation testbeds. In Bidot et al. (2003) and 


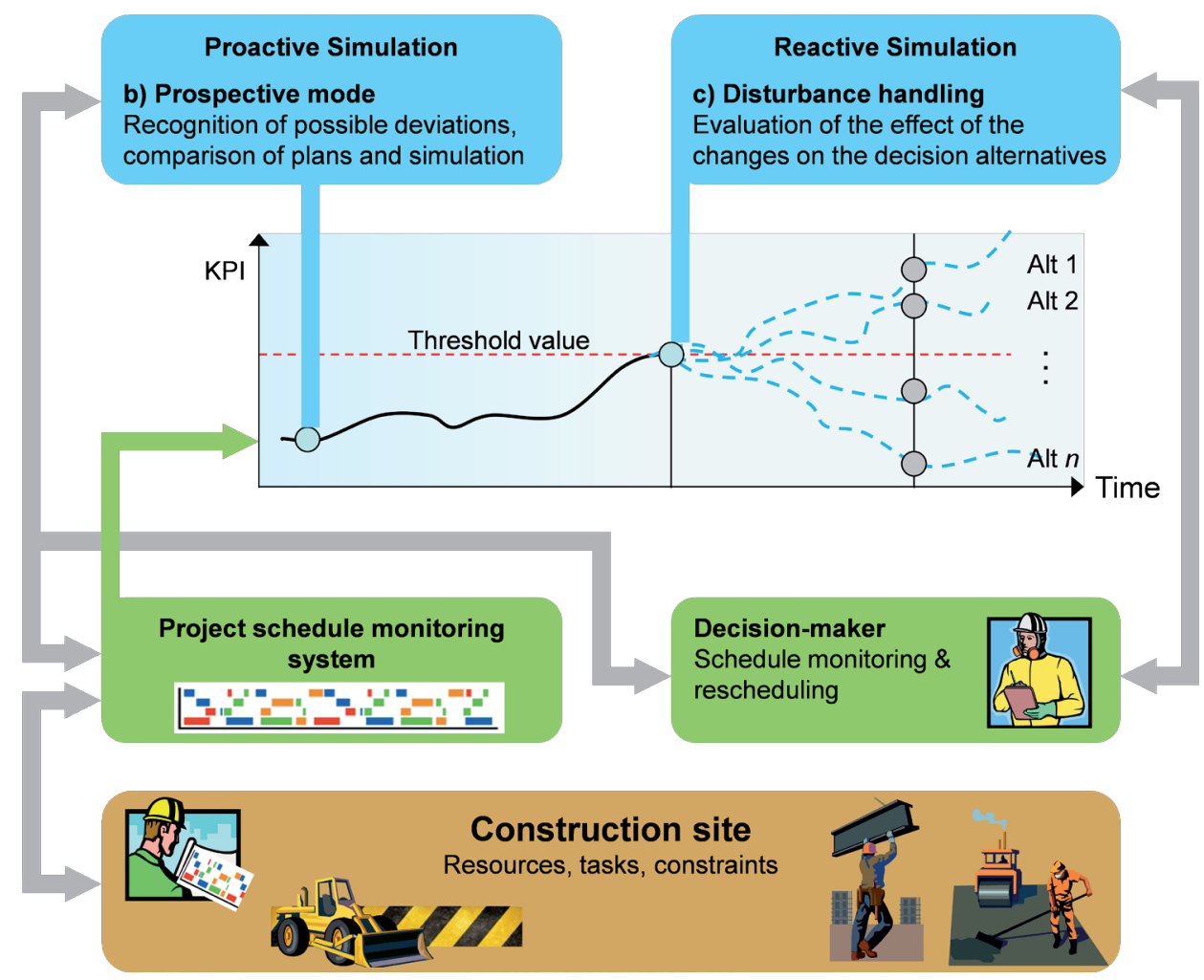

Fig. 1 Active disturbance handling realized by using reactive/proactive operation modes for simulation

in the paper of Cowling and Johansson (2002) the simulationbased execution of the calculated schedules is introduced considering uncertain activity durations in the form of probability distributions. Regarding the robustness and flexibility of tardiness and total flow-time in job-shops, several schedule repair methods are investigated in (Jensen, 2001), and an experiment is performed on a set of benchmark problems by executing schedules against simulated machine breakdowns. Sabuncuoglu et al. (2003) propose a simulation-based approach for testing the rescheduling methods in a dynamic and stochastic manufacturing system, applying uncertain processing times and machine breakdowns. In their approach the system consists of three components: simulation model, controller and scheduler. An interesting combination of deterministic and stochastic simulation is given by Honkomp et al. (1999). They describe a simulator for semi-continuous and batch processing manufacturing environments that can accept deterministic schedules and simulate both a deterministic and a stochastic realizations of the schedule. Running two versions of the simulation the authors compare the performance and robustness of the schedules.

\subsection{Model building and project schedule assessment 3.2.1 Requirements and functions}

As revealed in the previous literature review the proposed simulation module is suggested to be utilized as a component of a higher level system taking the role of the real production system or construction site. The reasons of connecting the scheduler to a discrete-event simulator are twofold. On the one hand, it serves as a benchmarking system for evaluating the schedules on a richer model. On the other hand, it covers the non-deterministic character of the real-life production environment. Additionally, in the scheduling phase it is expected that the statistical analysis of schedules should help to improve the robustness of execution and support the field manager during the calculation of further schedules. The main functions of the discrete-event simulator are as follows: it

- evaluates the robustness of weekly schedules against the uncertainties, performs sensitivity analysis of the schedules,

- helps in visualizing and verifying the results of a PPC system,

- supports rescheduling decisions.

The main functions (or operation modes) of the simulator in the proposed architecture (depicted in Fig. 1) are as follows:

- Off-line validation, sensitivity analysis of the schedules. Evaluation of the robustness of daily schedules prior to the execution against uncertainties, such as resource unavailability or job slipping. By this way, it can point out the resources which can endanger the realization of the daily schedule (not directly highlighted in Fig. 1).

- On-line, anticipatory recognition of deviations from the planned schedule by running the simulation parallel to the plant activities; and by using a look ahead function (supposing of keeping the sequences as planned), support of situation recognition (proactive operation mode, Fig. 1). 
- On-line analysis of the possible actions and minimization of the losses after a disturbance already occurred (reactive operation mode, Fig. 1).

The model structure in the simulator is the same for the three operation modes; however, the granulation (level of modelling details), time horizon, applied failure models and considered outputs depend on the purpose of the experiments.

In the on-line modes the simulation models represent various virtual mirrors of the construction sites and run parallel to the real environment, simulating also the future processes for a predefined short period. The performances of the predicted and the so far executed schedule are compared (highlighted as KPI, the performance measure of interest in Fig. 1).

The off-line operation mode refers to either a set of construction sites for interrelating projects (originally, in manufacturing environment factory or individual plants), while in the on-line modes the work of a site-level decision-maker is supported (Fig. 1). The main goal of the decision-maker is to ensure the completion of the daily/weekly schedule and if it is not possible to minimize the lateness of tasks. In case of occurred or predicted disturbances (as a consequence of changes), a decision has to be made, whether to intervene, or not. In the former case a rescheduling action has to be performed with a limited scope (in space and time) in correspondence to the sphere of authority of the decision-maker. The control action made in this rescheduling point incorporates the selection of the appropriate rescheduling policy and method (see. 2.2), which might be supported by the simulation-based analysis.

Based on previous studies summarized by Pfeiffer (2007), the proposed use of the system is the following.

In case of the performance measure of interest bypasses the threshold value (reactive operation mode), short-term, reactive simulations are initiated with the main aims of:

- analysing the influence of different rescheduling strategies on performance measures,

- exploring the rescheduling action with the smallest possible disturbance on the original schedule.

The way of the modification of the running schedule in the current case depends on the decision-maker, however, the selection of the right alternatives is reinforced by the system developed.

Based on the anticipatory recognition of deviations from the planned schedule by running the simulation parallel to the construction sites' activities (using the look ahead function and supposing the sequences are kept as planned), situation recognition is supported, moreover, deviations might be classified before they make real problems (proactive operation mode). This helps the decision making personnel in deciding whether to react, i.e., an intervention is required, or the recognized deviation has no or negligible effect on the schedule execution.
For more detailed description of the approach and more comprehensive results of the experiment, we refer to Pfeiffer (2007).

\subsubsection{Main phases of the simulation}

In order to meet all the requirements and achieve the desired functionality for a flexible simulation system, a so-called component-based simulation method has been developed by Pfeiffer et al. (2007). The simulation module and project scheduler have connections to the same project monitoring database. Resources, building plans, status information, i.e., directly and indirectly usable data are transformed exactly to the same form for all system components. Note that simulation relevant data (e.g. resource model, execution policies, process flow model) are stored locally in the simulation model.

Hereby, the complexity of integrating the simulation module into the system is significantly reduced. None the less, the common data tables ensure data integrity during the creation of the simulation model; moreover, the data-model serves as a basis for the more detailed construction process model. Running the simulation by applying the basic data tables results in a waste number of queries during the model run, reducing the simulation speed significantly. However, in order to ensure sufficient number of simulation replications for the evaluation of a short-term production schedule, the total response time should be minimized. In order to resolve the above two contradictory objectives an exhaustive data pre-processing phase is included in the simulation process.

Data preparation is carried out before the overall simulation. The redundant data storage in the simulation model is compensated by the advantage of the shorter response time. Modelling real production systems frequently brings up the problem of handling hundreds of resources in a simulation model. Having the modelling objects in hand, which were created on the base of the conceptual model, in our architecture the simulation model is created automatically based on the pre-processed data (phase b). The automatic generation of the model is followed by initialization (phase c). There, besides classical parameter settings, the procedure involves the generation of input parameter specific model components (entities such as products, operators). Contrary to the previous phase, this one is carried out for each replication. The simulation runs are repeated until the required number of replications is obtained (phase d). Each replication is a terminating, non-transient simulation run. In the last phase, the schedule is evaluated by using the evaluation criteria and the results of the evaluation process are interpreted by the decision-maker (e.g., site manager) who is responsible for taking the necessary actions.

\subsubsection{Novel model of the production execution process}

Regarding resource modelling of the designated production system (e.g. flow/job-shop model), a resource model class 
library was developed and applied for all the resource instances (Pfeiffer et al., 2007).

In construction industry, however, this model has to be adapted to the requirements. Namely, construction projects are similar to make-to-order customized production cases, where each final "product" is highly complex and unique (e.g., ship building, machine construction), in contrast to mass production (e.g., consumer goods, electronic components).

Thus, classes are pre-programmed component objects in the simulation, consisting of a generalized model of the resource, a built-in execution policy as well as the process flow of activities. Tasks represent the activities on particular parts (either a geographical location where material is used or a component required to be assembled), together with the assigned resources. As a main principle, the simulator should play back the schedule without changing the optimized sequence of the tasks, keeping the precedence order other tasks (see Table 2) but considering the calculated start times of the activities. Therefore, as a new solution, an ordered queue of the tasks is built up in front of each scheduled resource (TaskObject in Fig. 2), and the tasks to be processed are forwarded into these queues.

Table 2 Example for precedence constraints and assembled parts

\begin{tabular}{llll}
\hline Task ID & Designation & Precedes & $\begin{array}{l}\text { Proc. duration [day] } \\
\text { mean value / std. } \\
\text { deviation }\end{array}$ \\
\hline T1 & Constr. of abutment A & - & $15 / 7.5$ \\
T2 & Construction of pier B & T1 & $20 / 10$ \\
T3 & Construction of pier C & T1 & $22 / 11$ \\
T4 & Construction of pier D & T2, T3 & $20 / 10$ \\
T5 & Constr. of abutment E & T4 & $15 / 7.5$ \\
T6 & Construction of beam F & T3 & $8 / 4$ \\
T7 & Construction of beam G & T5 & $7 / 3.5$ \\
T8 & Construction of beam H & T3, T6 & $12 / 6$ \\
T9 & Construction of beam I & T7, T8 & $14 / 7$ \\
\hline
\end{tabular}

Each task has a list of the TaskObjects to be visited during the construction process, according to its process flow (building plan, originally routing) and the project schedule.

Arriving tasks at the resources are processed in the simulation as follows (see also Fig. 2):

- Tasks waiting for processing are stored in the input buffers of the resources (main resources at the site, e.g., crane) always sorted by their starting times. Each task in the schedule contains one of these TaskObjects, which, at the initialisation phase of the simulation, are distributed to the input buffer of the resources.

- The first TaskObject in the input buffer queue reserves the first position of the input buffer on the resource. This ensures that the designated resource is reserved for the designated task.

- If there is a TaskObject in the input buffer of the resource, which becomes ready at the moment, the setup process (preparation activity) will be immediately started (regarding the task represented by the TaskObject). Setup has to be started also when the part itself has not arrived yet at the TaskObject. In this case, there is no event generated by the arrival of the part for the simulation, however, because of the first criterion enlisted above, it is not allowed to start the setup process based on the calculated starting times. The proposed solution is to start the setup process, but freeze it immediately, before requesting the additional resources. It will be restarted only if the simulation time equals the planned starting time of the setup process.
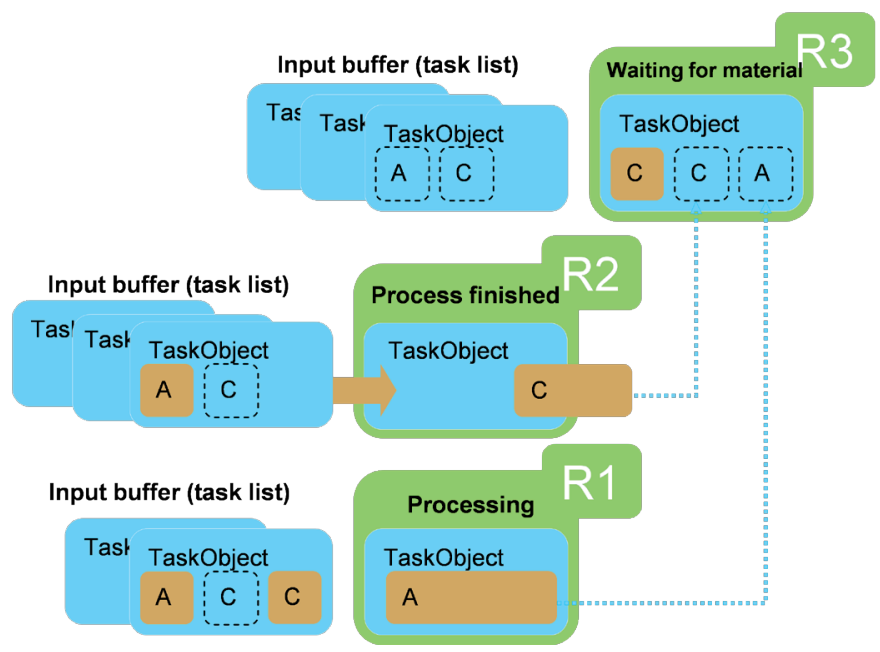

Fig. 2 Object-oriented model of the execution of the pre-calculated project schedule in the simulator, by applying the TaskObject structure.

- In order to start the task at least one additional resource (e.g., operator, truck, excavator) is needed with the designated service.

- If the setup process has finished and - in case it is an assembly process - all the required parts (component type) are already in the input buffer, then the main process can be initiated (e.g., crane elevates the concrete mixed to the defined level).

- Before the parts are reallocated to the resources, the processing times of the tasks are set, according to the specified attributes of the part objects. The processing of the parts is realized on two resource objects. For the first one no additional resource is necessary, while, for the second, it is mandatory to have at least one additional resource (see fourth criteria). In this case, the processing time of the first resource is calculated as the total resource time minus the additional resource time.

- If no free additional resource is available then the process cannot be started. 


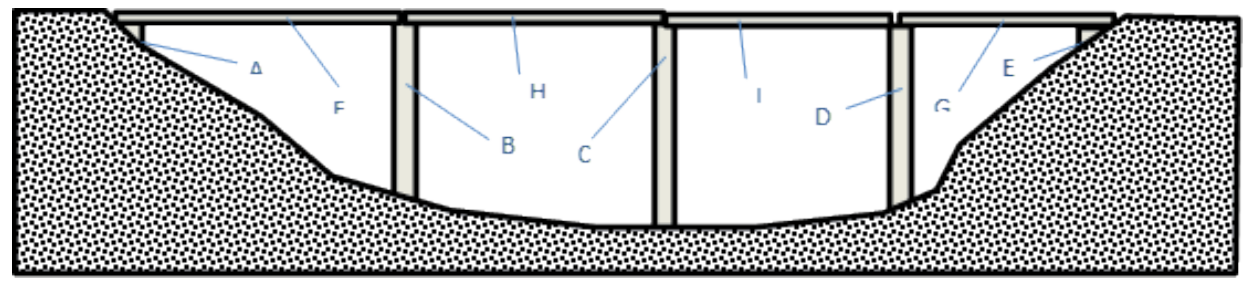

Fig. 3 Bridge building example for simulation modelling.

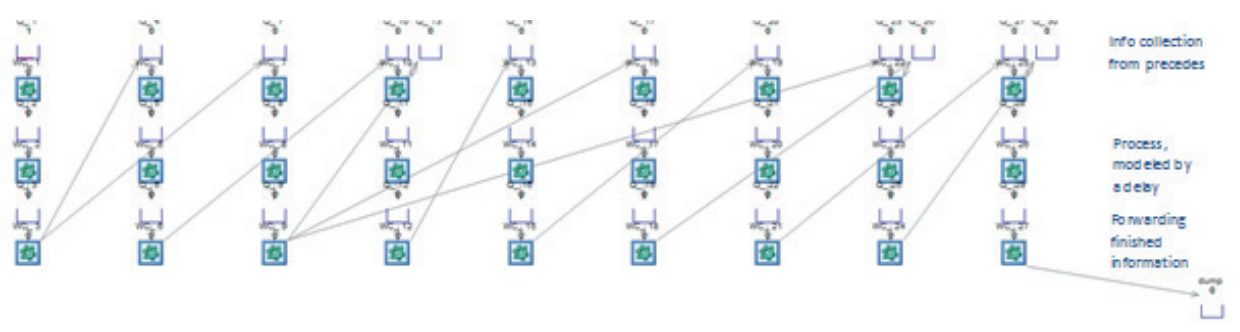

Fig. 4 Simulation model of the example process.

- After processing, parts are reallocated to the input buffer of the next resource.

\section{Simulation example}

For the above model a simulation example was developed using a standard Simul8 software (see: Simul8 Corporation, 2012). During the development aspects of creating a general tool was set as a priority. The simulation doesn't include initially the process structure it will be set up automatically using an internal code. Input is given using a process data table, like shown as Table 2. This specific example correspond to a bridge building project, in which a bridge with three piers are constructed. Planned sequence of the components' construction is given in Table 2, the components designation in Fig. 3.

The automatically generated Simul8 model's structure is shown in Fig. 4. The processes are drawn by the software from left to right. Each process is composed of three parts. The top part collects finished signals of precedes. The middle part implements the process itself using a single time delay from Table 2. The lower part is responsible for sending the ready signal to the consequent processes. During the tests 50 runs have been carried out with random variables from Table 2, where time mean values and deviations are used for normal distribution. Simulation runs resulted for the throughput time 93 days mean value and 10.02 days standard deviation.

\section{Conclusions}

This paper described comprehensively general and specific features of construction processes and relations to the dynamic scheduling problems known from manufacturing. It concluded that simulation support would be an effective tool to solve this problem. As reactive simulations may start from varying initial conditions automatic generation of the simulation model can be an advantage. This capability has been demonstrated using a Simul8 simulation software.

\section{Acknowledgement}

This research has been supported by the NFÜ (NDA National Development Agency) under the grant name KTIA AIK_12-1-2013-0009 - “Építési folyamatok kutatása”, with a total budget of 419.904.851 HUF.

\section{References}

Aytug, H., Lawley, M. A., McKay, K., Mohan, S., Uzsoy, R. (2005) Executing production schedules in the face of uncertainties: A review and some future directions. European Journal of Operational Research. 161(1), pp. 86-110. DOI: 10.1016/j.ejor.2003.08.027

Banks, J. (1998) Handbook of Simulation, Principles, Methodology, Advances, Application and Practice. John Wiley \& Sons Inc. 1998.

Bidot, J., Laborie, P., Beck, J. C., Vidal, T. (2003) Using simulation for execution monitoring and on-line rescheduling with uncertain durations. In: Proceedings of the ICAPS'03 Workshop on Plan Execution, Trento, Italy. 2003. DOI: 10.3182/20060517-3-FR-2903.0031

Cowling, P., Johansson, M. (2002) Using real time information for effective dynamic scheduling. European Journal of Operational Research. 139(2), pp. 230-244. DOI: 10.1016/S0377-2217(01)00355-1

Hao, Q., Shen, W., Neelamkavil, J., Thomas, R. (2008) Change management in construction projects. In: Proceedings of the CIB W78 25th International Conference on Information Technology: Improving the Management of Construction Projects Through IT Adopation. Santiago, Chile, 15-17 July 2008, pp. 387-396. DOI: 10.1109/CSCWD.2008.4536948

Hinze, J. (2011) Construction Planning and Scheduling. 4th Edition, Prentice Hall, New York. 2011. DOI: 10.1201/9781420041217.ch2

Honkomp, S. J., Mockus, L., Reklaitis, G. V. (1999) A framework for schedule evaluation with processing uncertainty. Computers and Chemical Engineering. 23(4-5), pp. 595-609. DOI: 10.1016/S0098-1354(98)00296-8

Jensen, T. M. (2001) Improving robustness and flexibility of tardiness and total flow-time job shops using robustness measures. Applied Soft Computing. 1(1), pp. 35-52. DOI: 10.1016/S1568-4946(01)00005-9

Kempf, K., Uzsoy, R., Smith, S., Gary, K. (2000) Evaluation and comparison of production schedules. Computers in Industry. 42(2-3), pp. 203-220. DOI: 10.1016/S0166-3615(99)00071-8

Law, A., Kelton, D. (2000) Simulation Modelling and Analysis. McGraw-Hill, New York. 2000. DOI: 10.1177/003754978203900606 
Motawa, I. A., Anumba, C. J., Lee, S., Peña-Mora, F. (2007) An integrated system for change management in construction. Automation in Construction. 16(3), pp. 368-377. DOI: 10.1016/j.autcon.2006.07.005

O’Reilly, J. J., Lilegdon, W. R. (1999) Introduction to FACTOR/AIM. In: Proceedings of the 1999 Winter Simulation Conference. pp. 201-207. DOI: $10.1145 / 324138.324200$

Pinedo, M. (2002) Scheduling: theory, algorithms and systems. Prentice Hall, NJ, USA. 2002.

Pfeiffer, A. (2007) Novel Methods for Decision Support in Production Planning and Control. PhD Thesis, Budapest University of Technology and Economics, Budapest, Hungary. URL: http://hdl.handle.net/10890/680 [Accessed: 4th September 2015]

Pfeiffer, A., Kádár, B., Monostori, L. (2007) Stability-oriented evaluation of rescheduling strategies by using simulation. Computers in Industry. 58(7), pp. 630-643. DOI: 10.1016/j.compind.2007.05.009
Rabelo, L., Helal, M., Jones, A., Min, J., Son, Y. J., Deshmukh, A. (2003) A hybrid approach to manufacturing enterprise simulation. In: Proceedings of the 2003 Winter Simulation Conference. pp. 1125-1133.

DOI: 10.1109/WSC.2003.1261541

Sabuncuoglu, I., Kizilisik, O. M. (2003) Reactive scheduling in a dynamic and stochastic FMS environment. International Journal of Production Research. 41(17), pp. 4211-4231. DOI: 10.1080/0020754031000149202

SIMUL8 Corporation (2012) SIMUL8. [Online]. Available from: http://www. simul8.com/ [Accessed: 11th December 2012]

Smith, S. F. (1994) OPIS: A methodology and architecture for reactive scheduling. In: Zweben, M., Fox, M. (ed.) Intelligent scheduling, Chapter 4. Morgan Kaufmann, San Francisco, USA. 1994. 